\title{
Escribir textos argumentativos para mejorar su comprensión
}

\author{
Joaquim Dolz
}

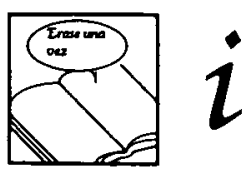

Como indica el título de este artículo, en él se abordará la relación entre la escritura y la lectura a propósito de las modificaciones que provoca la enseñanza de la primera sobre la segunda en un tipo de discurso particular : la argumentación. En una primera parte, se presentará brevemente la problemática de la interacción lectura-escritura en el campo de la didáctica de la lengua explicitándose en quésentido la escritura aparece como una de las estrategias para mejorar la comprensión. En la segunda parte, se introducen los seis elementos esenciales en la enseñanza de la argumentación. En la tercera y última parte, se aportan los resultados de una experiencia sobre la enseñanza de ese tipo de discurso, realizada en Ginebra (Suiza), con alumnos de 11-12 años.

\section{¿ESCRIBIR TEXTOS PUEDE CONTRIBUIR A FACILITAR LA LECTURA?}

Las relaciones entre la lectura y la escritura constituyen hoy en día un tema de interés tanto en el terreno escolar, como en el de la investigación científica. ¿Es posible en nuestra sociedad desenvolverse sin un dominio relativamente elevado del lenguaje escrito?

Los debates actuales sobre el fracaso escolar y el iletrismo (Besse et al., 1992) han puesto en evidencia que las exigencias de la sociedad en materia de lectura y de escritura son cada vez mayores. Sabemos perfectamente que esta sociedad excluye a todo aquel que no es capaz de leer y escribir textos diversos que van desde la simple nota para describir un itinerario, a la carta administrativa para solicitar una indemnización o argumentar una decisión.

Además, el dominio de la lectura y de la escritura está considerado por el profesorado como fundamental en todas las disciplinas. Para poder continuar sus estudios, el alumno deberá comprender un documento histórico, una explicación en un manual de física, la consigna de un problema de matemáticas, etc. Y, al mismo tiempo, deberá ser capaz de exponer por escrito una serie de saberes, presentar y comentar otro texto, argumentar su punto de vista sobre un tema, etc. Leer y escribir, es decir comprender y producir textos, no se consideran únicamente como fines en sí, sino también como medios para acceder a una escolaridad más larga. 
En ese marco, son cada vez más importantes las trabajos de reflexión y de investigación que analizan, por un lado, las características y los procesos movilizados por ambas actividades (las relaciones entre la lectura y la escritura desde un punto de vista estático) y, por otro lado, los efectos de una actividad sobre la otra (las interacciones entre lectura y escritura desde un punto de vista dinámico). En efecto, la lectura y la escritura son dos actividades que se apoyan constantemente la una sobre la otra, incluso en las primeras etapas del aprendizaje del lenguaje escrito.

Desde un punto de vista histórico y social, la lectura ha sido considerada como más importante que la escritura. Sólo en epocas relativamente recientes, la escritura de textos se convierte en un objetivo escolar al mismo nivel que la lectura (Chartier y Hébrard,1994). Y aún así, el aprendizaje de esta última aparece siempre como una etapa de preparación necesaria para abordar con un mínimo de bases el aprendizaje de la escritura. Socialmente, por otra parte, no saber leer está considerado aún hoy en día como más grave que no saber escribir.

Los esfuerzos de innovación didáctica de los últimos años han dejado de considerar la lectura y la escritura como actividades totalmente separadas. Se estudian los procesos de aprendizaje simultáneamente, tomando en consideración las complejas relaciones entre la comprensión y la producción del lenguaje escrito. Se elaboran cada vez más nuevas estrategias de enseñanza que presentan una visión integrada de la lectura y de la escritura. Esta tendencia hacia la unificación de las actividades lecturaescritura se ha visto acompañada de una reflexión sobre las diversidad textual. Cada vez son más los autores que critican la hipótesis de una competencia ligüística general que permita la comprensión o la producción del lenguaje. Las nuevas tendencias (Dolz, Bronckart y Pasquier, 1994) hablan de un conjunto de capacidades que pueden variar según se adopte la posición de lector o de escritor. Estas capacidades no son probablemente las mismas según las situaciones de comunicación y las características de los textos.

Si consideramos las interacciones entre lectura y escritura en el campo de la enseñanza, observaremos que las primeras concepciones de la enseñanza de la escritura la presentaban como pura copia de un modelo. Por lo tanto,

- la lectura es indispensable para el aprendizaje de la escritura;

- la lectura es la actividad principal que permite el desarrollo de la escritura.

La escritura es sin embargo una actividad mucho más compleja que la simple copia de un modelo. Exige la puesta en práctica de multiples habilidades que el escritor debe gestionar simultaneamente: comprender la tarea, representarse la situación de comunicación, elaborar los contenidos temáticos, planificar globalmente el texto, asegurar la cohesión del texto, utilizar un léxico adecuado, tomar en cuenta las reglas ortográficas y gramaticales, etc.

Si en algunos manuales de finales del siglo pasado y principios del actual, la lectura y la observación de ciertos géneros textuales (correspondencia privada y profesional, cuentos morales, descripciones y relatos de experiencias vividas, principalmente) aparecen ya como actividades fundamentales de preparación a la composición, la enseñanza metódica y directa de diferentes formas de textualidad, asociadas a los principales géneros textuales y a las condiciones sociales que determinan su uso en nuestra sociedad, ha exigido la realización de numerosas reformas a lo largo del presente siglo.

Descomponer los problemas de escritura relativos a un género textual y posteriormente preparar talleres y ejercicios para cada uno de esos problemas en orden a construir unidades o secuencias didácticas que mejoren la expresión escrita del alumno tal es una novedad que se ha desarrollado a partir de las últimas reformas.

Los nuevos métodos de enseñanza de la escritura no sólo se interesan por las influencia que la lectura puede tener sobre la escritura sino también por la forma 
cómo la segunda puede convertirse en una de las estrategias posibles de aprendizaje de la lectura.

Se habla de estrategias de lectura para mostrar el carácter flexible y dinámico de las actividades del lector cuando trata de construir la significación de un texto. Por extensión, algunos autores (Solé, 1993) hablan de estrategias de aprendizaje de la lectura para evocar las diferentes posibilidades en el aprendizaje de una lectura eficaz y controlada. La enseñanza de dichas estrategias persigue fundamentalmente la creación y el desarrollo de mecanismos de autorregulación en el alumno. Entre las estrategias de aprendizaje de la lectura podemos destacar:

- formular cuestiones sobre el texto leido;

- activar los propios conocimientos sobre el tema desarrollado en el texto;

- establecer inferencias entre las informaciones del texto;

- utilizar las macro-reglas (Kintsch y Van Dijk, 1976) : selección de las ideas principales, generalización, integración, etc.

Una de las maneras de enriquecer la problemática de las estrategias de de la lectura consiste en formular la hipótesis siguiente: pasar por la escritura constituye también una de las estrategias posibles para desarrollar la comprension de un texto. La escritura permite a los alumnos tomar conciencia de ciertas dimensiones lingüístico-discursivas que no siempre son tomadas en cuenta por los lectores precarios o en fase de aprendizaje de la lectura y que, por ello, constituyen un obstáculo para la comprensión de un texto.

Para ayudar a los alumnos a comprender mejor ciertas características asociadas a un género o a un tipo de discurso, en particular algunas de las características más complejas del discurso argumentativo, propongo la inversión de la secuencia dominante de la enseñanza tradicional que presenta la lectura de textos como una condición previa que permite y facilita la escritura «comprender y sólo luego producir». He utilizado, por lo tanto, una secuencia didáctica en la que dominan las actividades de producción de textos para observar su efecto sobre la comprensión.

En ese tipo de interacción donde las relaciones entre la escritura y la lectura se presentan de manera disociada, el grado cero consistiría en copiar un texto para entenderlo mejor (sabemos, por ejemplo, que Marx copió de su puño y letra algunos textos de Spinoza para captar mejor su significación). Tomar notas y resumir suponen un participación mayor y más activa de la parte del lector ya que implican la transformación del texto leido, la utilización «externa» de macro-reglas y la capacidad de reformular lingüísticamente el texto de base. En fín, pasar por actividades de escritura de textos del mismo tipo aparece como una estrategia de introspección para descubrir los mecanismos que constituyen un obstaculo en la comprensión de un texto particular. Cabe destacar que la escritura convoca siempre lectura o la relectura del texto que se está escribiendo y todo ello a un ritmo generalmente más lento que el de la escritura, lo que puede facilitar la toma de conciencia de algunas de las características del texto que dificultan la lectura.

\section{ELEMENTOS ESENCIALES DE LA ENSEÑANZA DE LA ARGUMENTACIÓN}

El discurso argumentativo es una actividad verbal específica cuyo aprendizaje está determinado por el contexto social y las intervenciones escolares. La escuela debería ser el lugar por excelencia de su aprendizaje para permitir el desarrollo de unas capacidades mínimas y la construcción de una base cultural común sobre la 
argumentación para todos los alumnos. Desde mi punto de vista, seis son los elementos a tomar en cuenta para desarrollar una enseñanza sistémica de la argumentación.

\section{Las situaciones de argumentación}

Toda argumentación se organiza a partir de una controversia, un desacuerdo, una polémica sobre un tema. Imaginemos, por ejemplo, una discusión en un centro escolar sobre la necesidad o no de proponer sistemáticamente deberes escolares para casa. Vemos inmediatamente que un tema como el que acabo de citar puede suponer un conflicto de intereses entre los padres, los profesores y los alumnos e incluso entre los propios profesores según sea su concepción de la enseñanza.

El argumentador, ya sea un alumno de $6^{\circ}$ de primaria o su profesor, adopta una posición sobre el tema (a favor o en contra la obligatoriedad de los deberes) y toma la responsablidad de defenderla o bien trata de construir su propia opinión en el curso de la discusión.

Generalmente, el objeto de una argumentación no es la verdad o la falsedad de una aserción sino el carácter más o menos verosímil o probable de la misma. Dicho de otra manera, una argumentación toma como objeto opiniones, actitudes o comportamientos discutibles. En el caso de nuestro ejemplo, tanto sugerir la generalización como proponer la pohibición de los deberes escolares son opiniones que se prestan a una discusión y no verdades evidentes.

Aunque las opiniones sean discutibles, el objetivo perseguido por el argumentador consiste en valorar esas opiniones, ya sea para crear (o crease) un nuevo sistema de convicciones, ya sea para atraer al otro hacia su propio punto de vista o para tratar de modificar su opinión. Se suele decir que el objetivo es convencer al destinatario cuando se utiliza sobretodo la razón y persuadir cuando se trata de obtener su adhesión con el sentimiento.

El destinatario de la argumentación sirve de elemento de regulación del discurso, en la medida en que no podemos modificar su actitud sin conocer su posición y analizar sus intereses. Si los alumnos que pretenden abolir los deberes a domicilio conocen los argumentos del sector del profesorado favorable a la obligatoriedad de los deberes, pueden tomarlos en cuenta para tratar de modificar la posición defendida por ese sector.

El lugar social condiciona el papel que juegan tanto el argumentador como el destinatario. En el caso de nuestro ejemplo, la escuela determina los roles sociales de los diferentes actores y condiciona sus posiciones (hablar sobre los deberes como alumno no es lo mismo que hacerlo como profesor).

Los géneros argumentativos dependen en gran medida del lugar social donde se producen (textos jurídicos, editoriales, sermones, lemas publicitarios, panfleto político, etc.).

\section{La estructura de base de los argumentos}

Un argumento está constituido al menos por dos proposiciones :

a) la opinión explicita que se quiere apoyar (conclusión: «los deberes no sirven para nada»);

b) las razones que sirven de justificación o de apoyo de esa opinión ( "porque los bacemos de mala gana, a toda prisa y luego nadie los corrige»). 
Generalmente, la proposición b presenta una serie de hechos o convicciones compartidas, o consideradas por el argumentador como aceptables para el destinatario, orientadas a dar mayor verosimilitud a la nueva opinión que se intenta comunicar con la proposición a.

Los autores que estudian la estructura de base o la secuencia prototípica de la argumentación se inspiran fundamentalmente en el esquema argumentativo de Toulmin (1958), primer autor en analizar la organización interna de los argumentos para asegurar su coherencia. Ep este mismo monográfico, Jean-Michel Adam presena sus propias hipótesis sobre la estructura elemental de la argumentación y analiza los componentes esenciales de la misma, dentro de una teoría general de las secuencias prototípicas textuales; Dominique Guy Brassart, por su parte, examina y discute el interés que presenta explotar esa estructura para enseñar a argumentar.

\section{Las operaciones específicas de la argumentación}

Una cosa es analizar las características estructurales de un argumento otra cosa distinta es tomar en cuenta los actos que intervienen en la producción de una argumentación.

La operación central de toda argumentación es la operación de apoyo argumentativo, operación que consiste en utilizar un segmento del discurso para dar soporte, justificar o acreditar el contenido de otro segmento del mismo discurso (Apothéloz y Mieville, 1989). La operación de apoyo argumentativo se manifiesta cuando un alumno presenta una serie de razones para justificar su posición sobre los deberes escolares.

La operación de negación argumentativa y de refutación, por su parte, consiste en rebatir la posición del adversario contestandola globalemente ( «no estoy de acuerdo con ciertos alumnos cuando dicen...") o en utilizar las propias razones del adversario para llegar a una conclusión opuesta ( A Aunque se bagan de mala gana, los deberes son esenciales para poder seguir correctamente esta asignatura»), lo que se llama contraargumentar. Es una operación que supone distanciarse de su propia opinión y tomar en consideración la opinión del otro.

Por último, la operación de negociaciación consiste en tomar en cuenta la posición del destinatario o del adversario para llegar a un compromiso.

\section{Las estrategias y los procedimientos retóricos}

La retórica clásica y la nueva retórica ha inventariado una serie de estrategias y procedimientos que no deben confundirse con las secuencias prototípicas. Algunos autores proponen organizar una enseñanza metódica para mejorar las capacidades del lector sobre contenidos como las figuras retóricas, los tipos de argumentos, la utilización de los tópicos, la ironía, la contradicción, las ejemplificaciones y otros procedimientos de inducción, etc. Se trata de permitir a cada uno la comprensión de los principales procedimientos que intervienen en la persuasión. La descomposición del razonamiento y las formas discursivas puestas en evidencia por la nueva retórica de Perelman y Olbrechts-Tyteca (1988) se aplican sobre todo como técnicas de interpretación de discursos diversos (jurídicos, publicitarios, filosoficos, políticos, etc.). Sin querer volver a una enseñanza tradicional de la retórica, suprimida desde el siglo XIX, creo que la didáctica de la lengua puede enriquecerse con sus aportaciones. 
Las unidades lingüísticas

Entre las marcas y los recursos lingüísticos más característicos del discurso argumentativo cabe destacar los siguientes:

a) las formas personales para asumir una opinión ( «Yopersonalmente pienso que...», "Me parece que...»);

b) las formulas para introducir citaciones ( «Según $X, \ldots »)$; los verbos de opinión (neutros: «X afima que... »; para desvalorizar: "X pretende... insinua...»);

c) los organizadores textuales (enunciados metalingüísticos para anunciar el plan : "Examinaré diferentes aspectos de la polémica. En primer lugar,... En segundo lugar,...»; organizadores de causa : "ya que», "puesto que», y de consecuencia : «por lo tanto»; etc.);

d) las modalidades del enunciado (expresiones de certeza : «estoy convencido que...», «seguramente...»; expresiones de probabilidad «parece ser que...», «probablemente...»;

e) las restricciones ( $a$ menos que... », excepto si...»);

f) formulas concesivas: "reconozco que...pero», "tengo que admitir... sin embargo»; etc.

\section{La planificación}

No existe una única forma de planificar globalmente un texto argumentativo sino diferentes posibilidades que pueden ser examinadas de manera crítica por los alumnos. Los argumentos se organizan en función de los objetivos que se quiere conseguir, de las características del destinatario a quien queremos convencer y de la tesis que se quiere defender. Se trata pues de enseñar a componer un texto adaptado a la situación de argumentación según un plan coherente y eficaz.

\section{UNA INVESTIGACIÓN SOBRE LA COMPRENSIÓN DE TEXTOS ARGUMENTATIVOS}

\section{Presentación de la experiencia}

\section{Situación que originó la investigación}

En el curso de una investigación que animo desde hace algunos años sobre la enseñanza precoz de la argumentación (Dolz, 1994; Dolz y Pasquier, 1994; Dolz, 1995), los alumnos de una clase de 6. ${ }^{\circ}$ de primaria de la ciudad de Ginebra $-11 / 12$ años - decidieron redactar, por iniciativa propia, una serie de cartas personales dirigidas al presidente de la Confederación Helvética, la mayor autoridad política suiza. En dichas cartas, hacían referencia al problema de uno de sus camaradas de origen extranjero, cuya familia, candidata al asilo político, acababa de recibir una orden de expulsión del país. Por ello, los alumnos suplicaban al presidente que la anulara y que permitiera a su compañero continuar en Suiza, al menos hasta el final del curso académico. La respuesta no se hizo esperar y, en ella, el presidente desarrollaba una argumentación para que los alumnos comprendieran y aceptaran las razones que habían conducido a las autoridades cantonales ginebrinas a expulsar a la familia de su camarada. 
La carta del presidente era una argumentación compleja y presentaba un número importante de formulas concesivas, destinadas a felicitar a los alumnos por su solidaridad, pero justificaba con una serie de razones la decisión de las autoridades cantonales. El principal argumento del presidente consistía en hacerles saber que incluso la más alta autoridad no está por encima de las leyes que rigen el asilo político en Suiza y, por ello, no puede permitirse ignorarlas o anularlas.

A pesar de su decepción, cuando los alumnos recibieron la carta del presidente, no tuvieron ninguna dificultad particular para comprender la decisión y las justificaciones del presidente y sin embargo la carta, como ya he dicho, era relativamente compleja. Hice entonces la hipótesis de que, en ese caso concreto, dos factores contribuían a facilitar su comprensión. Por una parte, los alumnos de esa clase conocían la situación de comunicación que había provocado la carta del presidente (era una situción creada por inicitiva espontánea de los alumnos). Por otra parte, esos alumnos habían desarrollado cierto número de capacidades argumentativas gracias a la realización de una secuencia didáctica de 15 horas de duración, en el marco de la investigación en curso sobre la producción de textos argumentativos.

Comencé entonces una nueva investigación en la que quise, en primer lugar, analizar los problemas de comprensión de textos argumentativos, como la carta del presidente, por parte de otros alumnos de la misma edad que desconociesen la situación que la había originado y que, para comprenderla, tuviesen que recontruir dicha situación. En segundo lugar, me propuse estudiar el efecto de una enseñanza centrada en la producción de textos argumentativos sobre la comprensión de la carta del presidente.

\section{Las características de la carta del presidente}

La carta que los alumnos debían leer presenta una serie de problemas relativos a tres dimensiones contextuales que me parecen fundamentales en la construción de la significación de un texto argumentativo : la intertextualidad, la polifonía y el dialogismo.

Desde el punto de vista de la intertextualidad, la carta es una respuesta a otras cartas. Forma parte de un intercambio epistolar comenzado cuando las autoridades cantonales ordenaron por escrito la expulsión de una familia de candidatos al asilo político y continuado por los alumnos de una clase cuando tomaron conocimiento de esa misiva y decidieron, a su vez, escribir al presidente de la confederación. La carta del presidente no puede comprenderse sin relación a los textos que la preceden.

Desde el punto de vista de la palifonía, en la carta del presidente se manifiestan diferentes voces. Se citan los autores de otras cartas: alumnos, profesores, familia X, autoridades cantonales. Se evocan las leyes del país, las distintas autoridades encargadas de aplicarlas, los pueblos confrontados a los mismos problemas que Suiza, los pueblos confrontados a la persecución política. El autor del texto habla a título personal cuando habla de la emoción que ha sentido leyendo las cartas de los alumnos y se expresa como presidente cuando justifica la decisión que «le exige su cargo».

Además la carta comporta un dialogismo mucho más profundo que el simple encadenamientos de cuestiones y respuestas y que se manifiesta cuando el autor retoma los argumentos de los alumnos y anticipa las reacciones que su respuesta negativa pueden provocar.

Por otra parte, la carta del presidente respeta la forma convencional de la correspondencia oficial pero articula globalmente los argumentos y los contraargumentos evitando la aserción directa de la respuesta negativa a la demanda de los alumnos. El lector debe inferir la posición del argumentador de no anular la expul- 
sión gracias a los argumentos y a los contraargumentos utilizados y, todo ello, a pesar de las concesiones y las modalizaciones introducidas para alabar la iniciativa de los alumnos.

En fin, la carta comporta una serie de características lexis y lingüístico-discursivas típicas del discurso argumentativo que no podré analizar aquí.

La reconstrucción de la situación de comunicación constituye una condición necesaria para la comprensión global de la carta. Si el lector de la misma no es su destinatario, reconstruir la situación de comunicación no es sólo una condición necesaria para dar un significado a las informaciones locales (sobre las condiciones de la familia afectada por la orden de expulsión, etc.), sino también para establecer una relación entre ellas y hacerse una representación global de la inteción comunicativa del autor.

\section{Dispositivo de la investigación}

La investigación en cuestión ha sido realizada en dos clases paralelas de una escuela del cantón de Ginebra, la primera constituye el grupo de control y la segunda el grupo experimental, siendo la variable independiente la secuencia didáctica.

Para asegurar un minimo de frablidad a la comparación, he elegido dos grupos de alumnos del mismo centro escolar que han obtenido en un test de comprensión de textos escritos (lectura de un dosier informativo sobre Europa) resultados similares.

Los alumnos de los dos grupos leyeron la carta del presidente y respondieron luego a las preguntas de un cuestionario. El grupo experimental realizó la misma tarea después de 15 horas de actividades centradas en la producción de textos argumentativos (la secuencia didáctica).

\section{El cuestionario de lectura}

En los dos casos, el enseñante facilita la tarea de los alumnos proponiéndoles un cuestionario que orienta la lectura de la carta y que ha sido elaborado para verificar el nivel de comprensión de la misma. Dicho cuestionario permite analizar y contrastar las dimensiones siguientes:

a) la reconstrucción de la situación de comunicación que provocó la redacción de la carta;

b) la identificación de la controversia que sirve de base de la argumentación y de la posición del argumentador en relación al problema discutido;

c) la organización de los argumentos en el texto;

d) la comprensión de ciertas marcas lingüísticas argumentativas; en particular, la comprensión de las fórmulas utilizadas como estrategias persuasivas y concesivas.

\section{La secuencia didáctica}

La secuencia didáctica experimentada (Dolz y Pasquier, 1993) está constituída por diez talleres (de hora y media de enseñanza diaria):

Taller 1.- Reconocer un texto argumentativo.

Taller 2.- Analizar las características de una situación de argumentación. 
Taller 3.- Clasificar argumentos.

Taller 4.- Dar su opinión y defenderla. Tomar en cuenta la posición del otro.

Taller 5.- Desarrollar argumentos. Usar los organizadores textuales de causa y de conclusión. Usar las expresiones de certeza y de probabilidad.

Taller 6.- Oponerse y formular objeciones.

Taller 7.- Contestar la opinión de una autoridad.

Taller 8.- Negociar. Usar las formulas concesivas y de cortesía.

Taller 9.- Planificar globalmente el texto siguiendo distintos circuitos.

Taller 10.- Elaborar pautas de control para la revisión y la escritura.

La primera versión de esta secuencia didáctica fue creada para mejorar la escritura de textos argumentativos trabajando en cuatro direciones distintas :

1) poniendo en contacto a los alumnos con situaciones argumentativas distintas para puedan captar la relación entre los parámetros de las situciones de interacción y las estrategias argumentativas utilizadas;

2) preparándolos para elabọrar distintos tipos de argumentos y contraargumentos;

3) facilitando el paso de la simple oposición a los argumentos del otro a la coordinación de puntos de vista y a la negociación de un compromiso;

4) practicando ciertas estrategias lingüísticas características de la argumentación.

\section{Hipótesis}

Enunciadas de manera sintética, las hipótesis de esta investigación son las siguientes:

1. Ciertas actividades que contribuyen a la comprensión de la carta del presidente son inaccesibles para gran parte de los alumnos de 11-12 años que no han trabajado nunca de manera metódica la argumentación. Las principales dificultades son las siguientes:

- reconstruir de manera precisa la situación de argumentación;

- identificar la posición del argumentador a propósito de la controversia;

- restablecer el orden de los argumentos a partir de la reformulación de los mismos;

- interpretar las fórmulas concesivas y las estrategias persuasivas.

2. En relación a esas cuatro dificultades espero mejorar los resultados en el grupo experimental gracias a la realización de la secuencia didáctica.

\section{Análisis de los resultados}

Los resultados del grupo control me han permitido observar el grado de comprensión de la carta de alumnos que nunca trabajaron sistemáticamente la argumentación en la escuela. De la comparación de los resultados entre el grupo experimental y el grupo de control se desprenden una serie de indicaciones relativas a los efectos de la secuencia didáctica sobre la comprensión de la carta.

\section{Reconstruir la situación de comunicación}

La mayor parte de los alumnos del grupo de control y del grupo experimental son capaces de identificar el texto como una carta que «responde a una demanda preci- 
sa» y que "presenta una serie de argumentos para convencer». Sin embargo, el $43 \%$ de los alumnos del grupo de control piensa que «se trata de una carta escrita por un especialista que presenta informaciones sobre Rumanía (país de origen de la familia afectada) y el $29 \%$ de los alumnos del mismo grupo piensan que el destinatario de la carta es «la familia $X » y$ no los alumnos de la escuela $Y$ ».

La imprecisión de la representación del papel del autor de la carta y del destinatario de la misma en el grupo de control se confirma cuando observamos que sólo el $62 \%$ de los alumnos atribuye el cargo de presidente de la Confederación al firmante de la misma (información explícita en la cabecera de la carta). Igualmente, aunque la mayoría es capaz de decir que la carta se dirige a escolares, sólo el $28 \%$ precisan que estos son los alumnos de una clase de la escuela $Y$, información que también aparece en la cabecera de la carta. Los alumnos que siguieron la secuencia didáctica obtuvieron resultados significativamente superiores a los del grupo de control en la identificación precisa del destinatario y del rol social del autor de la carta.

Una parte de los alumnos del grupo de control manifiesta dificultades en la reconstrucción precisa de la situación de interacción en la que la carta fue producida. Algunos de ellos no localizan las informaciones que se encuentran fuera del cuerpo de la carta y no establecen relaciones entre los diferentes índices. Como veremos a continuación, los alumnos que que no llegan a una buena representación de la situación de comunicación son aquellos que presentan mayores dificultades para identificar la posición del autor.

\section{Identificar la posición del argumentador}

La mayoría de los alumnos de los dos grupos identifica la controversia que sirve de base a la argumentación ( «la familia X tiene que volver a su país y quisiera poder quedarse en Suiza»). Pero, cuando se les pregunta si el autor del texto está de acuerdo con los destinatarios de la carta, gran parte de los alumnos del grupo de control (58\%) contesta «si». La incomprensión de la posición del argumentador queda confirmada por otras cuatro cuestiones que van en el mismo sentido. Vemos, a traves de las respuestas, que una parte importante de los alumnos del grupo de control piensa que el autor del texto «autoriza a la familia $X$ a quedarse en Suiza».

Quisiera también hacer observar que existe una fuerte correlación entre las diferentes respuestas relativas al mismo sujeto (phi entre.59 y .73) y entre esas cuestiones y las relativas a la reconstrucción de la situación de comunicación (phi entre .39 y .48). Existe, por lo tanto, una relación entre la comprensión de la situación de comunicación y la posición del argumentador.

En los alumnos que siguieron la secuencia didáctica, la mejor representación de la situación de comunicación va de par con una identificación mucho más clara de la posición del argumentador. Casi todos los alumnos del grupo experimental afirman, sin contradecirse en el cuestionario, que la respuesta del presidente es negativa.

\section{Restablecer el orden de los argumentos}

Restablecer el orden de los argumentos reformulados es una actividad inaccesible para la mayoría de los alumnos del grupo de control (24\% de respuestas totalmente satisfactorias contra $78 \%$ del grupo experimental). Para restablecer el orden correctamente, los alumnos tienen que establecer una correspondencia entre los argumentos de la carca y su reformulación en el cuestionario y, luego, deben ordenarlos según aparecen en la carta. Un ejercicio de ese tipo exige una apropiación global 
del razonamiento del autor del texto, una gran flexibilidad para identificar los indices textuales y una capacidad de síntesis de cada uno de los párrafos. Los alumnos que consiguen ordenar los argumentos reformulados son aquellos que obtienen mejores resultados en el conjunto de la prueba.

\section{Interpretar las concesiones $y$ las estrategias argumentativas}

Las concesiones y las estrategias persuasivas utilizadas por el autor no siempre son interpretadas correctamente por los alumnos (o como lo haría un lector con experiencia). Los lectores precarios interpretan como una aceptación de la demanda de los alumnos de la clase $Y$ las numerosas modalizaciones y concesiones del autor del tipo : "Os aseguro que comprendo perfectamente los sentimientos que habeis experimentado al enteraros de que vuestro comañero iba a ser expulsado. Comprendo también que vuestro mayor deseo sería que puediera quedarse en Suiza. Pero...». Los enunciados introducidos por el autor para hacer comprender diplomáticamente su decisión, hacen que interpreten las sutilezas como una aceptación de la solicitud. En este punto, las diferencias entre el grupo experimental y el grupo de control son claras. Los alumnos del primer grupo sustituyen sin dificultad las formulas concesivas por otras similares, la mayoría de los alumnos del grupo de control son incapaces de hacerlo.

No podré por falta de espacio comentar con detalle los resultados de todas las preguntas del cuestionario. Para dar una imagen general tomando todas las cuestiones de comprensión confundidas, señalaré que el $81 \%$ de las respuestas de los alumnos del grupo experimental son justas, mientras que el rendimiento medio del grupo de control es sólo del $55 \%$.

\section{Consideraciones finales}

Para terminar, quisiera hacer una serie de consideraciones destinadas a poner en relación algunos resultados de esta investigación con algunas pistas para mejorar la enseñanza de la argumentación.

Los resultados del cuestionario revelan algunos de los principales problemas de comprensión que me parecen específicos al texto argumentativo:

1. Los alumnos de esta edad no evaluan siempre de manera oportuna la situación de interacción social en la que se produce la controversia, lo que constituye un obstaculo para identificar la posición del argumentador.

2. Cuando no identifican la posición del argumentador, les cuesta captar la orientación de los argumentos y de los contraargumentos.

3. No comprenden siempre la articulación entre los argumentos.

4. A menudo, no hacen la distinción entre las formulas concesivas y persuasivas y la decisión misma del argumentador. Las primeras son utilizadas por el argumentador como una estrategia para de reconocimiento del destinatario, con la intención de suavizar la decisión tomada. Los alumnos las confunden con la decisión.

Acabo de señalar cuatro problemas de comprensión que me parecen fundamentales y específicos del texto argumentativo. Es probable que el alumno encontrará ese mismo tipo de dificultades cuando lea un editorial de prensa, una texto de opinión, un informe destinado a tomar una decisión, etc. La tarea para el especialista en didáctica de la lengua y para los profesores consiste en imaginar proyectos de lectura y secuencias didácticas que tomen en consideración esas dificultades.

Como lo muestran los buenos resultados de mi experiencia, las actividades de producción de textos y la toma de conciencia que se realiza durante los talleres de 
una secuencia didáctica parecen proporcionar al alumno nuevos instrumentos de regulación de la lectura, asegurando un mayor nivel de eficacia. Y ello, para los cuatro problemas de escritura que acabo de evocar. En otros términos, los talleres de la secuencia didáctica facilitan el desarrollo de capacidades de construcción de la significación ausentes en la mayoría de los alumnos del grupo de control. Gracias a esas capacidades argumentativas, los alumnos consiguen una lectura más flexible y distanciada; es decir, una mayor autonomía lectora. Se puede sugerir, desde ese punto de vista, el paso por actividades con una fuerte proporción de producción escrita para mejorar la lectura.

Aunque los resultados de mi experiencia deben ser considerados con prudencia ya que el grupo que participó es demasiado reducido, creo que se puede afirmar, sin lugar a dudas, que producir textos argumentativos ayuda a comprenderlos mejor.

\section{Notas}

1. Ver el balance de investigaciones reunidas en las Actas del coloquio Théodile-Crel.

\section{Referencias}

ADAM, J.M. (1981): «Votez Mir Rose, achetez Giscard» : analyses pragmatiques, Pratiques, 30, 73-98. ADAM, J.M. (1993): Les textes : types et prototypes, Nathan.

ANSCOMBRE, J.C. y DUCROT O. (1988): L'argumentation dans la langue, Bruxelles, Mardaga.

APOTHELOZ, D. y MiEvILle, P. (1985): Etude des représentations au moyen des organisations raisonnées et des objets de discours. Principes méthodologiques et exemple d'analyse, Travaux du Centre de Recherches Sémiologiques, 49, 57-70.

APOTHELOz D. y Mieville D. 1989: «Matériaux pour une étude des relations argumentatives». En Modeles du discours. Recherches actuelles en Suisse romande, Ch.. Rubattel éd., Berne, Peter Lang.

BoIsSINOT, A. y LASSERRE, M.M. (1989): Techniques du franģais : Lire-argumenter-rédiger, Paris, BertrandLacoste.

BoREL, M.-J. (1991): «Notes sur le raisonnement et ses types», Etudes de Lettres, 4-1991, Université de Lausanne.

BRASSART D. G. (1990): «Retour(s) sur «Mir rose» ou comment analyser et représenter le texte argumentatif (écrit)?", Argumentation, 4,

BRASSART, D.G. (1990): Le développement des capacités discursives chez l'enfant de 8 à 12 ans. Le discours argumentatif (étude didactique). Revue Française de Pédagogie, 90, 31-41.

BronCKART, J.P. et al. (1985): Le fonctionnement des discours, Neuchâtel: Delachaux \& Niestlé.

BRONCKART, J.P. (1987): Interactions, discours et significations, Langue franģaise, 74, 29-50.

DE PATER W.A. (1965): Les «Topiques» d'A ristote et la dialectique platonicienne. Méthodologie de la définition, Etudes Thomistes, Vol. X, Fribourg, St Paul.

DOLz, J. (1993): Ia argumentación, Cuadernos de Pedagogía, Monográfico Leer y escribir, 216, 68-70.

DoLz, J. (1993): La interacción de las actividades orales y escritas y la enseñanza de la argumentación, Comunicación y lenguaje, Monográfico sobre la lengua oral,26, 68-70.

DOLZ, J. (1995): Learning argumentative capacities : a study of the effects of a systematic and intensive teaching of argumentative discourse in 11-12 year old children, Argumentation.

DOLZ, J. y PASQUIER, A. (1993): Argumenter... pour convaincre: initiation aux textes argumentatifs. Cabiers du service du français, 31 , DIP, Genève.

Dolz, J. y PASQUIER, A. (1994): Enseignement de l'argumentation et retour sur le texte. Repères, 10, DIP, Genève.

DOLZ, J. PASQUIER, A. y BRONCKART, J.P. (1993): L'acquisition des discours : Emergence d'une compétence ou apprentissage de capacités langagières ? Etudes de linguistique appliquée, 89.

Ducrot O. y ANscombre J.-C. (1983): L'A rgumentation dans la langue, Bruxelles, Mardaga.

DuCROT O. (1980): Les Echelles argumentatives, Minuit, Paris.

GOLDER C. (1992): Argumenter : De la justification à la négociation, Archives de Psychologie, 60, 232, 3 24

GRIZE, J.B. (1981): L'argumentation : explication ou séduction. Linguistique et sémiologie,10, L'argumentation, Lyon, PUL.

KINTSCH W. y VAN DIJK T. A. (1978): Toward a model of text comprehension and production, Psychological Review, 85, 5, 363-394.

MOESCHLER, J. ( 985): Argumentation et conversation, Paris, Hatier. 
Perelman C. (1983): «Logique formelle et argumentation». En P. Bange et al. éds. : Logique, argumentation, conversation, Berna: Peter Lang.

Pereiman, C. y Olbrechts-TytecA, L. (1988). Traité de l'argumentation, Bruxelles, Editions de l'Université de Bruxelles.

PLANTIN Ch. (1990): Essais sur l'argumentation, Paris, Kimé.

SOLÉ, I. (1993): Lectura y estrategias de aprendizaje,Cuadernos de Pedagogía, Monográfico Leer y escribir, 216, 68-70.

Toulmin S.E. (1958): The Uses of argument, Cambridge, Cambridge University Press.

VAN DIJK T. A. (1980): Macrostructures. An Interdisciplinary Study of Global Structures in Discourse, Interaction and Cognition, Hillsdale, Lawrence Erlbaum Ass.

VIEHWEGER, D. (1993): Savoir illocutoire et inmterprétation des textes. En: M. Charolles, S. Fisher \& J. Jayez (eds.) Le discours : représentations et interprétations, Presses Universitaires de Nancy.

VIEHWEGER, D. (1984): Le Dire et le dit, Paris, Minuit.

\section{Escribir textos argumentativos para mejorar su comprensión Joaquim Dolz CL\&E, 1995, 26}

Resumen: Este artículo presenta una experiencia realizada en Ginebra con niños de 11-12 años. La experiencia consiste principalmente en hacer escribir textos argumentativos para mejorar su lectura y su comprensión e ilustra un tipo particular de intervención didáctica, de carácter interaccionista, que busca principalmente crear una relación entre dos formas de trabajo: dotar al alumno de medios para analizar las situaciones sociales en las que se produce (o se lee) un texto argumentativo y favorecer la apropiación del valor socio-enunciativo de algunas de sus características lingüísticas específicas. Las actividades de aprendizaje se organizan en una secuencia didácrica que comporta diez talleres sobre diferentes dimensiones de la argumentación. El autor demuestra que el trabajo realizado en producción contribuye a desarrollar las capacidades de interpretación de otro texto del mismo tipo: mejor representación de la situación de interacción, de la posición del argumentador, mejor identificación de las concesiones y de las estrategias persuasivas.

Datos sobre el autor: Joaquim Dolz, Doctor en Ciencias de la educación, es miembro del equipo de didáctica de las lenguas de la Universidad de Ginebra (Suiza). Sus investigaciones actuales versan sobre la didáctica del texto escrito, en particular sobre la enseñanza de la argumentación y del relato de enigma.

Dirección: Faculté de Psychologie et Sciences de L'Education, Université de Genève, 11, route de Drize, 1227 Carouge (Suisse). Ver el balance de investigaciones reunidas en las Actas del coloquio Théodile-Crel presentadas por Reuter (1994).

(C) PERMISOS PARA CITAR O REPRODUCIR EN OTRAS FUENTES: Se pueden citar libremente hasta 500 palabras. Para reproducir una porción de texto mayor, figuras o ilustraciones, se deberá pedir permiso por escrito a la revista, especificando el uso al que se destina el texto. En todos los casos, se deberá citar el copyright de $C L \& E$. En el caso de artículos o textos que hayan sido a su vez reproducidos en $C L \& E$ los interesados deberán dirigirse tanto a los detentadores del copyright original como a $C L \& E$, en el caso de que se quiera hacer uso de la traducción. FOTOCOPIAS: Para todo lo relacionado con el uso mediante fotocopia del material de esta revista, deberán dirigirse a: CEDRO, C/ José Marañón, 10, 3. Izda. Tel. 5941575 . Fax 4453567 